\title{
Marseille et ses natures: permeabilites spatiales, segmentations sociales
}

\section{Marseille e suas naturezas: permeabilidades espaciais, segmentações sociais}

\section{Marseille and its Natures: Spacial Permeabilities, Social Segmentations}

\author{
Cécilia CLAEYS* \\ Jean-Noël CONSALES** \\ Carole BARTHÉLÉMY***
}

\begin{abstract}
RÉSUMÉ
Cet article propose une relecture de la question de la nature dans la ville à travers les paradigmes environnementaux émergeants questionnant l'ancienne dichotomie sauvage/artificiel. A partir d'une étude de cas, Marseille, ville méditerranéenne française, l'analyse interdisciplinaire articulant sociologie et géographie révèle comment les nouveaux modes de gestion de la nature urbaine et périurbaine (Parc National périurbain, trames vertes, jardins collectifs, parcs publics) interrogent les frontières spatiales entre sauvage et artificiel, tandis que les usages demeurent marqués par des formes de segmentations sociales persistantes.
\end{abstract}

Mots clés: nature dans la ville; interdisciplinarité sociologie/géographie; Marseille; Parc National des Calanques; trames vertes.

\section{RESUMO}

Este artigo propõe uma releitura da questão da natureza nas cidades por meio dos paradigmas ambientais emergentes que questionam a antiga dicotomia selvagem/artificial. A partir de um estudo de caso, Marseille, cidade mediterrânea francesa, a análise interdisciplinar, articulando sociologia e geografia, revela como os novos modos de gestão da natureza urbana e periurbana (Parque Nacional periurbano,

\footnotetext{
"Maître de Conférences em Sociologia. Laboratório Population Environnement Développement (População, Meio Ambiente e Desenvolvimento), UMR 151, AixMarseille Université. E-mail: cecilia.claeys@univmed.fr

"** Maitre de Conférences em Geografia. UMR TELEMME 6570, Aix-Marseille Université. E-mail: jean-noel.consales@univ-amu.fr

*** Maître de Conférences em Sociologia, Laboratório Population Environnement Développement (População, Meio Ambiente e Desenvolvimento), UMR 151, Aix-Marseille Université. E-mail: carole.barthelemy@univ-amu.fr
} 
corredores verdes, jardins coletivos, parques públicos) questionam as fronteiras espaciais entre o selvagem e o artificial, enquanto os usos continuam marcados por formas de segmentações sociais persistentes.

Palavras-chave: natureza nas cidades; interdisciplinaridade sociologia/geografia; Marseille; Parque Nacional das Calanques; corredores verdes.

\begin{abstract}
This paper proposes a re-reading of the question of the nature in the city through the emerging environmental paradigms questioning the old dichotomy wild / artificial. From a case study, Marseilles, a French Mediterranean city, interdisciplinary analysis articulating sociology and geography reveals how new modes of urban and suburban nature management (suburban National Park, greenways, community gardens, public parks) query the spatial boundaries between wild and artificial, while uses are still marked by persistent social segmentations.
\end{abstract}

Keywords: urban nature; interdisciplinary sociology/geography; Marseille; National Park of the Calanques; greenway.

\section{Introduction}

Si le rôle de la nature en ville a déjà une longue histoire dans nos sociétés occidentales, force est de constater qu'il fait l'objet depuis quelques années d'un renouveau, revisité à la lumière des paradigmes environnementaux émergeants (DUBOST; LIZÉE, 2003). Villes durables, trames vertes, parcs périurbains, gestion différenciée sont autant de vocables qui désignent de multiples initiatives visant à repenser, au nom de la protection de l'environnement, la frontière symbolique et/ou matérielle entre la ville et la nature. L'une des grandes tendances novatrices résiderait dans le passage d'une nature pensée en termes d'hygiénisme et de verdure à une nature souhaitée spontanée, sauvage ou bien encore, endémique. Un tel changement est porté par la vague du développement durable interrogeant la compatibilité entre densification urbaine et qualité des milieux de vie, qu'ils soient humains ou naturels (MATHIEU; GUERMOND, 2005). Il amène à discuter la pertinence des frontières traditionnelles opposant ville et nature, sauvage et domestique, rare et ordinaire, reconnaissant, sinon valorisant, voire même incitant les perméabilités au sein de ces dichotomies.

L'objet de notre propos est de faire dialoguer sociologie et géographie autour de cette nature urbaine dans une ville, Marseille, qui se prête très bien à l'exercice, pour différentes raisons. Marseille, tout d'abord, a la double réputation d'être pauvre en espaces verts "urbains" (parcs et jardins) et riche en espaces naturels "périurbains" arides (collines et Calanques). La ville fait actuellement l'objet d'un intérêt croissant quant à ses milieux naturels; Baptiste Lanaspeze parle à son sujet de "ville nature de demain" (2012). Les analyses sociologiques et géographiques permettent de saisir les fondements et les limites d'une telle réputation, à la lumière des caractères territoriaux du site et des processus sociohistoriques à l'origine de cet héritage physique et symbolique. Quel est l'état et l'étendue objectivable des espaces verts/naturels urbains et périurbains (première partie)? Ces héritages expliquent, en grande partie, les rapports contemporains à la nature, tant dans les formes urbaines que dans les pratiques des habitants. Quelles sont les pratiques ordinaires des Marseillais vis-à-vis de ces espaces urbains et périurbains (seconde partie)? Marseille accueille, enfin, deux projets en cours de réalisation, la mise en place d'une trame verte, d'une part et la création du Parc National des Calanques, d'autre part qui se révèlent particulièrement propices à l'observation des liens qui se tissent, entre cette ville et ses natures. Comment ces projets participent-ils peu ou prou d'une redéfinition du rapport entre les marseillais et leurs natures (troisième partie)?

Cet article s'appuie sur une lecture transversale de différents corpus de données spatiales et sociologiques constitués lors de plusieurs enquêtes de terrain réalisées 
dans le cadre de programmes de recherche nationaux. Il regroupe les éléments suivants:

- Une analyse spatiale des Espaces à Caractère de Nature (ECN, CLERGEAU, 2007), réalisée dans le cadre du programme PIRVE "Atlas Analytique de la Trame Verte à Marseille".

- Une enquête par questionnaires réalisée en 2009 et 2010 auprès des habitants de trois quartiers de la ville de Marseille (Quartier Sud, Centre et Nord), $(n=330)$ (CLAEYS, 2010).

- Une enquête par entretiens semi-directifs menées sur site en 2010 et 2011 auprès des usagers terrestres et marins du massif des Calanques $(n=92)$ réalisée dans le cadre du programme LITEAU "Caractérisation de la fréquentation littorale et détermination d'indicateurs de vulnérabilité écologique pour définir des modes de gestion durable - Cas de la bande littorale du projet de parc national des calanques" (TATONI, LE MIRE PECHEUX, 2010).

- Une enquête par entretiens semi-directifs menée auprès de dix gestionnaires et de douze agents d'entretiens des parcs publics de la ville $(n=22)$ (BOURDIL, 2010) réalisée dans le cadre de l'ANR Villes Durables "Trame verte urbaine".

- Une enquête au long cours (depuis 1999) sur les jardins collectifs marseillais fondée sur de l'observation participante et des entretiens semi-directifs réalisés auprès de la totalité des dirigeants des structures associatives de gestion et de plus d'adhérents jardiniers $(\mathrm{n}=200)(\mathrm{CON}-$ SALES, 2004)?

- L'observation directe des processus de concertation et de décision des gestionnaires de la nature urbaine et périurbaine de Marseille réalisée dans le cadre des programmes PIRVE et CDE "Un Parc National pour les Calanques de Marseille? Construction territoriale, forme de concertation et principes de légitimité" (DELDREVE; DEBOUBT, 2011).

\section{Héritages: l'eau et les collines, éléments structurants $\mathbf{d u}$ territoire marseillais}

Les relations qu'entretient Marseille à ses natures, sont inhérentes aux spécificités physiques d'un site dominé par deux éléments naturels forts:

- l'eau, marquée par la présence incontournable de la mer et celle plus discrète d'un fleuve côtier (l'Huveaune), d'une rivière (le Jarret) et de ruisseaux (notamment celui des Aygalades),

- les reliefs calcaires, faits de collines et de massifs, entre lesquels s'intercalent de rares espaces plans.

Eau et collines constituent les marqueurs dominants de l'identité territoriale marseillaise, et ce, jusque dans la légende de la fondation de la ville. ${ }^{1}$ Ces éléments naturels ont influencé vingt-six siècles d'une histoire urbaine riche, en servant autant d'ancrage à la cité phocéenne que de limites physiques à son territoire. Pendant plus de 2200 ans (jusqu'en 1666), Marseille reste cantonnée à son site originel, sur la rive nord de la anse du Lacydon (actuel Vieux Port) (figure ${ }^{\circ}{ }^{\circ} 1$ ). Face au soleil du sud et tournant le dos au mistral froid, elle règne sur un territoire circonscrit par la Méditerranée et par un amphithéâtre de collines et de massifs calcaires, que viennent uniquement percer le ruisseau des Aygalades, au nord, et plus amplement l'Huveaune, au sud-est (RONCAYOLO, 1996). Entre les enceintes artificielles de la ville et celles naturelles de son territoire, s'organise alors, autour de nombreux hameaux et villages ruraux, un vaste "terroir" agricole, indispensable à la pérennité de la cité.

De fait, à Marseille comme dans toutes les villes d'Europe occidentale, les rapports ville/nature se bornent pendant longtemps à la seule complémentarité ville/agriculture que sous-tendent les banlieues agricoles. Dans la première partie du XIX ${ }^{\mathrm{eme}}$ siècle, le territoire marseillais est le cadre d'une agriculture intensive, mobilisant environ 9000 hectares et 25000 personnes (D.D.A., 1971).

\footnotetext{
${ }^{1}$ La tradition veut, en effet, que celle-ci naisse, en 600 avant J.-C., lorsque Gyptis, la princesse de la peuplade ligure installée à l'intérieur des terres, choisit pour époux Protis, le chef des marins venus de Phocée en quête de nouvelles colonies, en lui tendant une coupe d'eau. Dans la symbolique, Marseille trouve donc ses origines dans l'union de la mer et de la colline, célébrée par le partage de l'eau.
} 


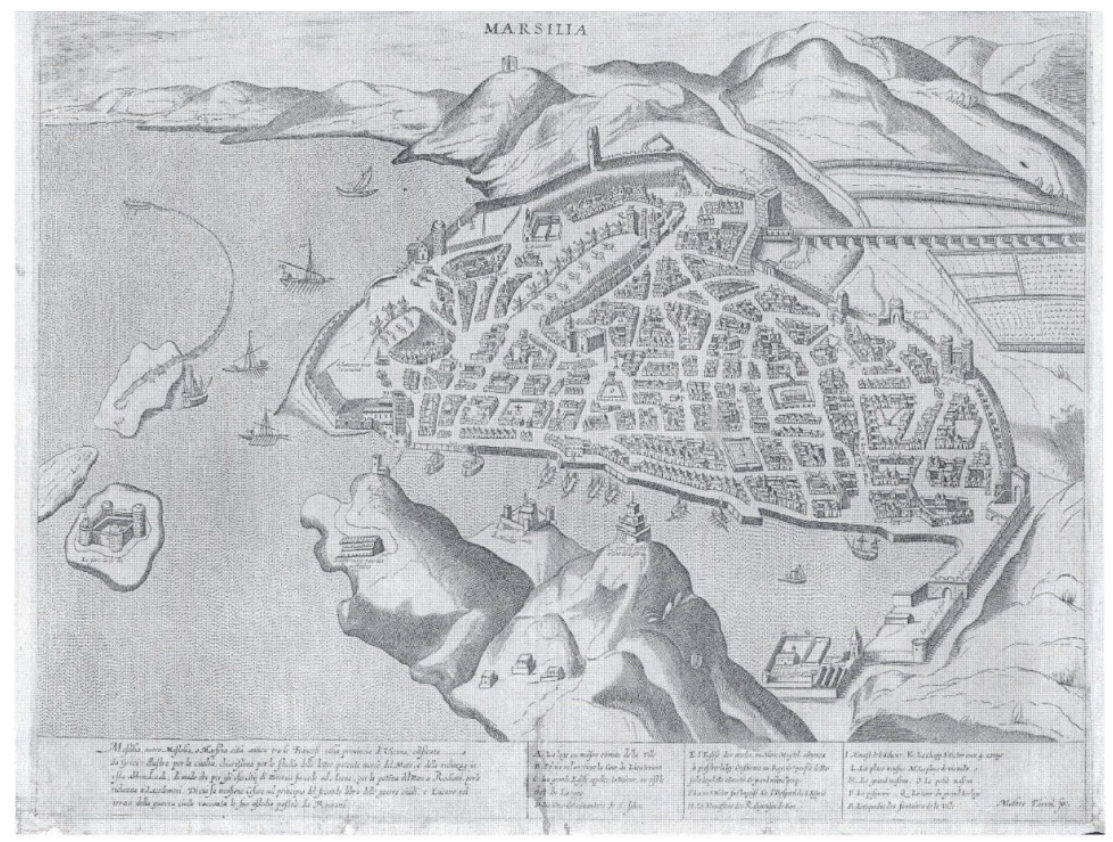

FIGURE 1 - Carte: XVI ${ }^{\mathrm{e}}$ siècle, Vue cavalière de la ville de Marseille et de son terroir vue vers le Nord par Mathéo Florini (Archives Municipales de Marseille).

Face à la rigueur du climat méditerranéen et de ses sécheresses estivales, cette activité se concentre alors le long de l'Huveaune et du Jarret.

Ce paysage agreste est néanmoins totalement redessiné avec l'édification du Canal de Marseille, en 1849, qui approvisionne depuis la ville en eau issue du massif montagneux des Alpes. Le terroir marseillais s'érige, dès lors, en véritable banlieue maraîchère et laitière, répondant aux besoins de la ville. La cité s'impose comme le moteur des initiatives agricoles. Les profits réalisés par les bourgeois urbains dans le commerce maritime et l'industrie sont le plus souvent réinvestis dans la terre, source essentielle de prestige. Ce processus se matérialise, dans le paysage rural marseillais, par un marqueur territorial incontournable: la bastide.

Spécificité territoriale marseillaise, la bastide se présente, du XV ${ }^{\text {ème }}$ au XIX ${ }^{\text {ème }}$ siècle comme composante paysagère majeure, restant pourtant cachée derrière de puissants murs, elle ne se révèle que par une vue surplombante (CHANCEL; BORRUEY, 1993; RIANI, 2000). Véritable gageure territoriale, mettant la ville à la campagne (MÉRY, 1860), la bastide s'érige, au fil du temps, en exemple, inspirant notamment le mouvement cabanonier des classes populaires. La bastide témoigne de la porosité des frontières entre la ville et la campagne, entre le naturel et l'urbain. Elle traduit surtout le rapport étroit qu'entretiennent les Marseillais avec leur terroir et la nature productive (VIARD, 1989).

Cette structuration traditionnelle du territoire marseillais est, cependant, bouleversée à partir du XIX ${ }^{\text {ème }}$ siècle. Sous les effets de la Révolution Industrielle, la cité phocéenne connaît, en effet, un essor sans précédent. L'extension du port et de ses zones industrielles, lui confère une ouverture prépondérante non seulement sur la Méditerranée, mais encore sur l'immense empire colonial français. Expansion et densification urbaines grignotent les terrains agricoles et les parcs et jardins de Bastides (RONCAYOLO, 1996). Ce processus d'urbanisation qui touche la citée phocéenne ne manque pas de susciter quelques vives inquiétudes. Face aux problèmes engendrés par la croissance massive de la population citadine et par l'étalement urbain qui en découle, Marseille s'ouvre à de nouvelles politiques d'aménagement parmi lesquelles des jardins publics. 
Certaines envisagent la nature comme ressource à la crise urbaine. En suivant modestement l'exemple hygiéniste du Paris du Second Empire d'Haussmann, les édiles municipaux conçoivent des projets d'aménagements paysagers et de jardins publics répondant à la volonté de la classe bourgeoise dominante de voir la "ville à la campagne" (LIMIDO, 2002).

De ce point de vue, la "nature urbaine" de Marseille, à l'instar des grandes villes françaises, est de prime abord caractérisée par une dominante de verdure, inféodée à la présence d'eau, de facto (sources et cours d'eau) ou d'irrigation (le canal de Marseille). Toutefois, un autre territoire marseillais échappe à cette représentation sociale dominante en Occident qui associe nature, verdure et eau. Le massif des Calanques situé entre Marseille et Cassis est en effet majoritairement constitué de milieux semi-arides qui ne donnent pas ou peu lieu à des mises en valeur agricole. Dans le massif des Calanques, étymologiquement "pentes abruptes", du provençal "calanca", les activités agropastorales traditionnelles sont éparses. Le ramassage du petit bois de chauffe, les fours à chaux et les charbonnières traditionnels accentuent l'aridité naturelle du site (MASSENA-GOURC, 1994). La façade maritime du massif abritent, nichés aux creux de profondes Calanques, des pêcheurs aux petits métiers occupant temporairement des habitats de fortune souvent sans droit ni titre, les cabanons (GONTIER, 1991).

Jusqu'au XIX ${ }^{\text {ème }}$, le massif des Calanques constitue un territoire en marge, d'un point de vue esthétique, social et économique. Difficilement accessible, le massif ne fait pas ou peu l'objet d'aménagements lourds. A partir du XIX ${ }^{\text {ème }}$ siècle, ce territoire et ses usages connaissent de visibles changements suivants deux axes antithétiques, caractéristiques de la modernité montante et de ses critiques. L'un relève de l'industrialisation de l'exploitation du site et l'autre appartient aux prémisses de la protection de la nature. Ainsi, au développement de fours à chaux industriels s'ajoute l'installation d'usines de fabrication de plomb, de soude et d'acides. Toutefois, l'activité florissante de ces productions industrielles est de court terme (CONSALÈS; GOIFFON, 2005). Et, avant même leur déclin, d'autres pratiques se posent sur les flans du massif marseillais, passant sans transition ou presque du prélèvement à visée productive à la protection à visée récréative. Dans la mouvance du Club
Alpin Français (C.A.F.) et de la mise en application des premiers textes juridiques explicitement en faveur de la conservation des milieux, notamment la loi du 4 avril 1882, dite loi de "restauration des terrains de montagne" le massif des Calanques est redéfini comme Haut-Lieu de nature (MASSENA-GOURC, 1994; DURBIANO, 2003). Ainsi, à travers la section marseillaise du C.A.F. et son transfuge local, l'association des excursionnistes marseillais, des membres de la bourgeoisie urbaine trouvent très tôt dans le massif des Calanques un territoire propice à la projection des redéfinitions esthétiques portées au niveau national par une élite intellectuelle inspirée par les savoirs naturalistes et hygiénistes prônant le grand air et la performance sportive. Cette requalification esthétique semble d'emblée traversée par une tension entre la sublimation du minéral, monument de nature avec lequel l'alpiniste fait corps et le désir de verdure qui prend la forme concrète de récurrentes pratiques de reboisement par les acteurs institutionnels (notamment l'Office National des Forêts) et associatifs (en particulier les scouts de France).

L'activisme de cette élite locale pionnière de la protection de la nature, ainsi que le relais pris à partir de la fin des années 1960 par les couches moyennes porteuses des préoccupations environnementales contemporaines contribuent à limiter les effets de la croissance urbaine sur ce territoire naturel. La ville vient lécher les flans les plus proches du massif, grignoter quelques vallons et absorber les anciens villages de pêcheurs sans toutefois atteindre le vaste cœur des Calanques. Car, hormis sur les massifs calcaires, le processus d'urbanisation entamé dès la Révolution Industrielle ne manque pas de se poursuivre durant les Trente Glorieuses, transformant la ceinture agricole en banlieue résidentielle. Celle-ci s'organise autour des nombreux noyaux villageois qui maillent ce territoire périphérique et utilise souvent, comme support foncier, les immenses domaines bastidaires facilement cédés par leurs propriétaires. Cependant, les chocs pétroliers successifs de 1973 et de 1979, ainsi que la crise du secteur industriel plongent la ville dans une profonde récession économique. Marseille ne satisfait plus totalement ses habitants, désormais en quête de qualité et d'authenticité des cadres de vie. Dans les campagnes périphériques, qu'elles soient intra-communales ou non, la périurbanisation se traduit principale- 
ment par une vague de constructions pavillonnaires. Ce sont alors les terres agricoles qui font les frais de cette évolution. Perçues comme une véritable réserve de terrains à bâtir, cette matrice agricole résiduelle, faite le plus souvent de friches et de délaissés végétalisés, sert, sur le territoire communal, de support au processus récent de densification périphérique, sous forme de pavillons et de petits collectifs en résidences fermées (CONSALÈS et al., 2012).

Que reste-t-il alors aujourd'hui, après deux siècles d'urbanisation intense, des relations traditionnelles entre Marseille et ses natures? Bien que plus complexes, celles-ci semblent perdurer, comme tend à le démontrer le document suivant (figure n. ${ }^{\circ} 2$ ).

Intitulée "Marseille en négatif", cette carte recense les principaux Espaces à Caractère de Nature de la commune, en faisant abstraction des éléments bâtis. Elle laisse, ainsi, apparaître quatre unités distinctes, déterminant des relations spécifiques de la ville à la nature (CONSALÈS et al., 2012). La Méditerranée, tout d'abord, immuable limite physique à l'ouest et au sud, conditionne des rapports dichotomiques, de part et d'autre du Vieux Port. En direction du sud, la mer se fait paysage et le littoral se pose en véritable gradient de naturalité. En direction du nord, la mer est fonctionnelle, servant de support à une activité portuaire qui dessine un trait de côte artificiel. Puis, sur le territoire communal, l'augmentation des nuances et des densités de vert, depuis le centre vers la périphérie, permet de mettre en exergue trois autres unités. La première d'entre elle est marquée par une faible densité de vert. Elle correspond à la frange littorale plus ou moins plane sur laquelle se sont développés les espaces centraux et péricentraux. Dans cette unité, les ECN semblent presque exclusivement composés de jardins publics (parcs et squares) ou privés (jardins en cœur d'îlots. La seconde unité est caractérisée par une augmentation manifeste de la densité du vert. Celle-ci s'inscrit sur l'ensemble de petites collines, de plateaux et de pentes qui forme les piémonts des massifs calcaires périphériques. C'est le domaine de l'ancienne banlieue agricole, devenue résidentielle durant les Trente Glorieuses. Ici, les ECN semblent essentiellement se composer de jardins privés (dans les cités d'habitat collectif et les lotissements pavillonnaires) et de fric hes, autrefois agricoles, recouvertes d'une végétation spontanée. La troisième et dernière unité terrestre est entièrement verte. Elle correspond aux massifs calcaires qui circoncissent le territoire marseillais. Au nord, les massifs de la Nerthe et de l'Etoile sont protégés par des outils de planification locaux, tandis qu'au sud le Parc National des Calanques vient d'être créé (avril 2012). La carte de "Marseille en négatif" permet donc de mettre en évidence des ensembles territoriaux distincts, en fonction des relations de Marseille à ses Natures.

\section{Usages entre partage et ségrégation: parcs, jardins et collines}

La nature urbaine à la fois proche, visible et accessible est celle offerte par les parcs et jardins publics. Ces derniers accueillent diverses pratiques urbaines de nature, ludiques ou sportives, isolées ou conviviales, ... (SANSOT, 1995). La conflictualité y est peu ou prou limitée par une structuration des différents espaces de loisirs. La spatialisation des pratiques est généralement induite par les aménagements existants. Parfois, c'est l'aménageur qui doit au contraire s'adapter aux résistances des usagers, comme ce fut le cas dans la réglementation de l'accès aux pelouses, finalement ouvertes bon gré mal gré aux turbulences footballistiques des jeunes marseillais (NIEL, 2009). Là, des grappes d'enfants à l'assaut des balançoires et des toboggans, ici des seniors préférant les bancs ombragés, et encore les sportifs foulant les chemins selon des itinéraires spécifiques, des contre-allées au parc, du parc au bord de mer. Ainsi, le parc public est une mise en scène du corps urbain. Dans cette ville littorale, le parc public s'entremêle volontiers avec l'espace balnéaire, telles les plages du Prado et leurs espaces verts, conquis sur la mer à partir de 1977 et ayant remporté un succès auprès des Marseillais au-delà des espérances des aménageurs (HÉRAT, 2010).

Les chiffres manquent, mais les observations directes sont sans appels: les parcs de Marseille sont soumis à un haut niveau de fréquentation. Pourtant les conflits d'usage demeurent limités ou tout au moins ne font pas l'objet d'une problématisation spécifique. La promiscuité, recherchée par les uns, subie par les autres, semble donner lieu à une relative acceptation parce qu'inhérente à la ville, par définition espace de concentration humaine. 


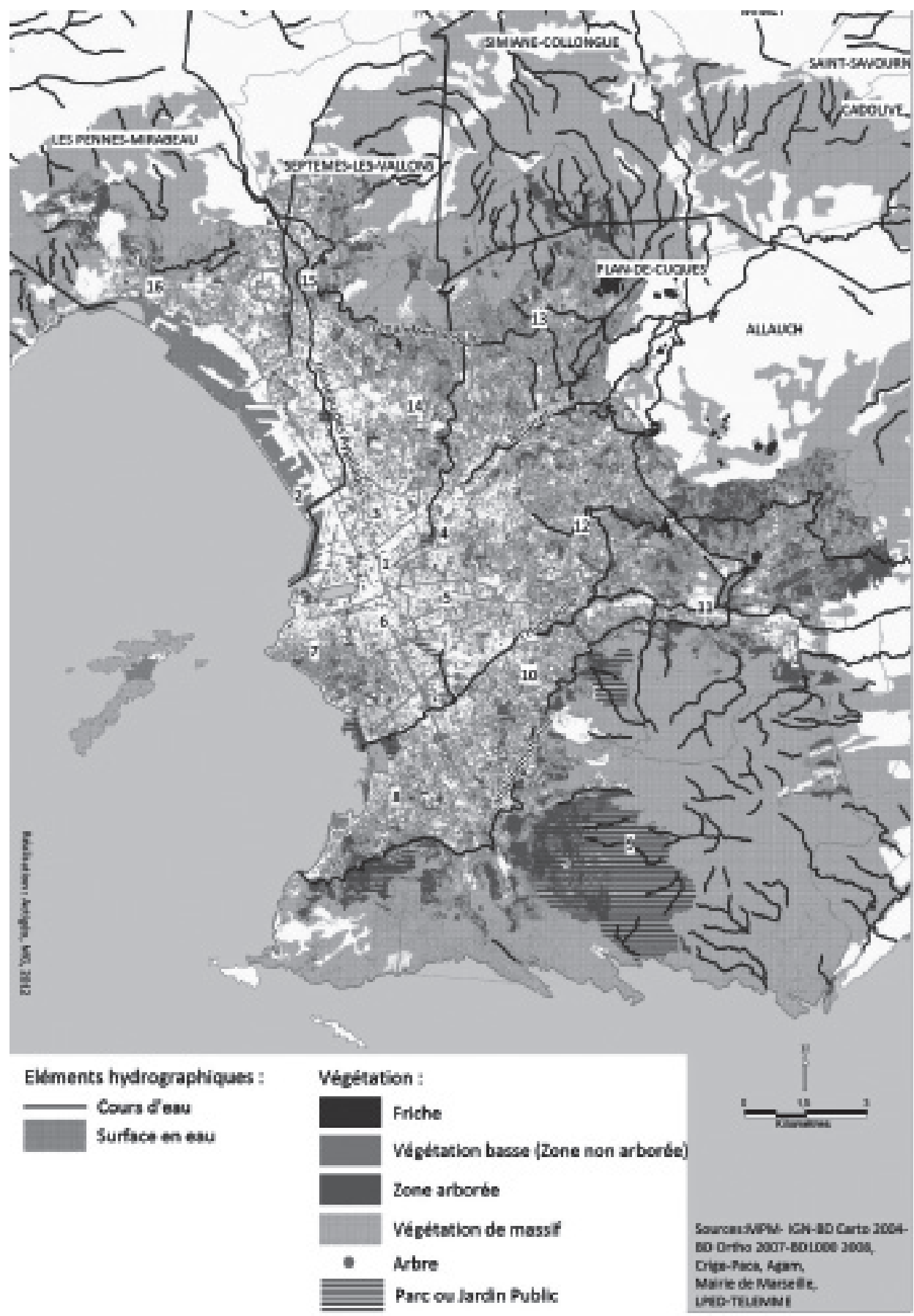

FIGURE 2 - Carte: Marseille en négatif. Auteur: Actigéo MG Atlas Analytique de la Trame Verte à Marseille. 
Au même moment, d'autres natures se cachent au creux de jardins privés, riches et populaires, ou restent invisibles, car mésestimées, sur les friches et les délaissés végétalisés. Loin de toute logique ostentatoire, le jardin privé participe, à Marseille, de la sphère de l'intime, de l'espace personnel, familial ou micro-social. Associé à l'habitat, il ne se donne pas à voir, restant coupé de l'extérieur par des clôtures ou des murs impénétrables, non sans liens avec la tradition bastidaire. Il se révèle, néanmoins, par une vue dominante tant au centre de la ville, où il s'immisce en cœur d'îlot, qu'en périphérie, où il structure les cités d'habitat collectif et les lotissements pavillonnaires fermés (Consalès et al. 2010). Illustration de cet état de fait, les quelques sites de jardins familiaux qui existent à Marseille demeurent peu connus et reconnus (Consalès, 2003), car complètement fermés à la ville et au paysage urbain. Souvent entretenus par des personnes âgées irréductiblement attachées à "leur morceau de campagne" (selon l'expression couramment employée par les jardiniers), ces collectifs de potagers s'entourent de hauts murs de protection, sciemment ou inconsciemment destinés à les protéger des malveillances citadines. Cette discrétion plus ou moins volontaire les rend non seulement invisibles mais encore impraticables pour les habitants des quartiers dans lesquels ils s'implantent. A l'intérieur de ces véritables enceintes vertes, les objectifs différenciées, voire contradictoires, des jardiniers tendent à générer des pratiques différentes, sinon divergentes. En fonction de l'évolution progressive du rapport à la nature nourricière, les logiques productives quantitatives et résidentielles traditionnellement associées aux jardins familiaux (Weber, 1998), se conjuguent de plus en plus avec des logiques productives qualitatives et naturelles, caractérisées par la montée en puissance d'un jardinage respectueux de l'environnement (CONSALÈS, 2008). Cette évolution ne va pas alors sans engendrer des conflits d'usages entre les jardiniers, difficilement régulée par les dirigeants associatifs. L'exemple de ces collectifs de potagers démontre donc qu'à Marseille, le jardin privé relève encore aujourd'hui véritablement du sens étymologique du mot jardin, consacrant celui-ci en tant qu'espace clos. Il est néanmoins affecté par la montée de la pensée environnementale qui tente de redéfinir les anciens modes de gestion de ces espaces traditionnels. A l'inverse, la myriade de friches et autres délaissés disséminés sur le territoire communal, au nord-est notamment, apparaissent comme des espaces végétalisés ouverts. Ils n'en demeurent pas moins invisibles, car ignorés en tant qu'Espaces à Caractère de Nature. Résultant d'une urbanisation périphérique à faible densité de bâti, ces friches à vocation agricole se distinguent, pourtant, quant à leur impact sur le maintien de la biodiversité urbaine et se présentent, par ailleurs, comme de réelles aménités paysagères, surtout au printemps lorsque la flore spontanée les transforment momentanément en jardins méditerranéens

Ainsi se côtoient en ville des natures domestiques et ré-ensauvagées, celle des parcs publics à la grégarité acceptée, des jardins cachés intimistes et des friches ouvertes à la visibilité sociale en devenir. A la fois proche et lointaine, une autre nature se déploie, celle des collines et des Calanques, oscillant elle aussi entre domestique et sauvage, entre grégarité et isolement.

A l'instar de bien d'autres espaces "naturels", les Calanques sont depuis plusieurs décennies le théâtre de la démocratisation des loisirs de nature et des dramaturgies produites par la diversification et l'intensification des activités propices aux conflits d'usages et de représentations. Dans un contexte d'augmentation régulière du temps libre (CORBIN, 1995; DUMAZEDIER; 1988) et de démocratisation relative des préoccupations environnementales, tous partagent le même désir de Calanques, comme échappatoire aux maux urbains. En revanche, chacun assouvit son désir de nature selon son habitus de classe, mais aussi générationnel, où l'ambivalence entre l'ordinaire, le familier et le remarquable rapproche tout autant qu'elle divise les usagers du site s'appropriant symboliquement et/ou matériellement les lieux. Dans ce contexte, la définition des Calanques comme espace de liberté est un leitmotiv, mais son sens et sa mise en pratique prend des formes différentes et parfois incompatibles, faisant s'opposer l'individuel et le collectif, l'intellectualisé et le ludique, le bruit et le silence, l'isolement (seul ou avec des membres choisis) et le grégaire, la nonchalance et la performance du corps...

Les usagers héritiers du CAF et des excursionnistes privilégient la contemplation érudite comme forme d'appropriation symbolique et se posent en faux contre toutes formes d'appropriation matérielle des 
Calanques, condamnant les prélèvements sur la nature (chasse, pêche, cueillette) et la forme d'habitat qui y est intiment lié, le cabanon. Pourtant, au moment même où se dessine un faussé marqué et durable entre ces deux grands types d'usagers du site (MASSENA-GOURC, 1994), l'opposition première qui les sépare, contemplation versus prélèvement devient ténue. Les cabanons de pêcheurs et de chasseurs d'antan sont en effet progressivement aménagés et transformés en lieu de villégiature. D'autres sont d'emblée construit à cet effet (GONTIER, 1991). Les "parties de cabanons", festivités populaires et masculines ont progressivement été élargies à des pratiques familiales. La part des produits de la chasse et de la pêche dans la consommation au cabanon s'amenuise pour disparaitre presque totalement parfois, au profit de denrées achetées en supermarchés acheminées sur place en voiture, bien loin du pittoresque périple du siècle dernier, en sabots ou espadrilles, à côté de l'âne bâté.

Aux héritiers directs des tous premiers cabanoniers viennent progressivement se greffer par jeux de réseaux d'interconnaissance et de recommandation d'autres familles locales, participant d'un processus de gentrification. Dès lors, l'opposition n'est plus entre contemplation versus prélèvement de la nature, mais davantage entre itinérance et sédentarité. La persistante opposition entre patrimoine naturel universel convoqué par les randonneurs et patrimoine culturel local mobilisé par les cabanoniers est toutefois transcendée par leur commun désir de préserver le massif des Calanques de l'industrie touristique et des investisseurs immobiliers. Ces derniers partagent aussi les mêmes références à la mer, au soleil et à la beauté du site (AZIBI; PARMASKYAN, 2006).

Au-delà de la diversité des loisirs ne serait-ce que terrestres $^{2}$ (marche, escalade, jogging, V.T.T., parapente, farniente, balnéaire, chasse, parties de cabanon,...), une même activité peut prendre des formes et des significations très variées selon les individus. La marche, activité terrestre dominante dans les Calanques (43\% des usagers (GIP CALANQUES, 2009)) est, à ce titre, particulièrement symptomatique. Groupes de jeunes gens turbulents issus des quartiers populaires proches ( $\mathrm{La}$
Cayolle), familles élargies faisant quelques pas digestifs, amoureux alanguis ou échangistes à l'affut, ${ }^{3}$ marcheurs amateurs ou randonneurs confirmés partagent tout autant qu'ils se disputent les mêmes portes d'entrée du massif.

Ces conflits d'usages et de représentation particulièrement exacerbés lors du processus de concertation (DELDREVE; DEBOUBT 2011; CLAEYS, 2011) mené dans le cadre de la création du parc national ne doivent toutefois pas cacher une persistante homogénéité sociale et culturelle parmi les usagers du site. Les conflits les plus visibles mettent en jeu des acteurs fortement présents dans l'espace des débats publics, mais ayant un poids démographique faible (chasseurs, pêcheurs, cabanoniers). Une telle distorsion entre visibilité culturelle et politique et poids démographique tient aux enjeux politiques locaux, nationaux et internationaux relatifs à ces pratiques spécifiques dans un contexte de restriction toujours croissante de ces dernières, au nom de la protection de la ressource. L'enquête par questionnaires réalisée auprès d'un échantillon de marseillais révèle en effet que la fréquentation du massif en général et sa fréquentation régulière en particulier, sont davantage le fait des individus appartenant aux couches moyennes et supérieures et bien dotés en capital culturel (figure n. ${ }^{\circ} 3$ ).

Notre enquête par entretiens semi-directifs menée sur site corrobore la prédominance des individus à fort capital culturel parmi les usagers des Calanques. Or, les quartiers urbains les plus proches des Calanques sont aussi parmi les plus aisés de la ville de Marseille, tandis que les banlieues populaires en sont les plus éloignées (figure n. ${ }^{\circ}$ 4). Ici, ségrégations sociales et spatiales se renforcent, forme exacerbée d'inégalités écologiques (DEBOUBT, 2010).

Ainsi, la fréquentation du massif témoignerait d'une diffusion d'une certaine forme d'appropriation de la nature esthétique, sportive et naturaliste qui trouverait dans les Calanques une expression aboutie. La massification de la fréquentation des Calanques relèverait moins d'une démocratisation des loisirs de nature mais plutôt de la diffusion d'une forme de rapport à la nature qui n'est pas démocratiquement partagée.

\footnotetext{
${ }^{2}$ Cet article fait le choix délibéré de rester centrée sur les activités terrestres. Un parallèle reste à faire avec la démocratisation des activités nautiques (plongée, plaisance, ...)

${ }^{3}$ Pratique essentiellement localisée au Montrose.
} 


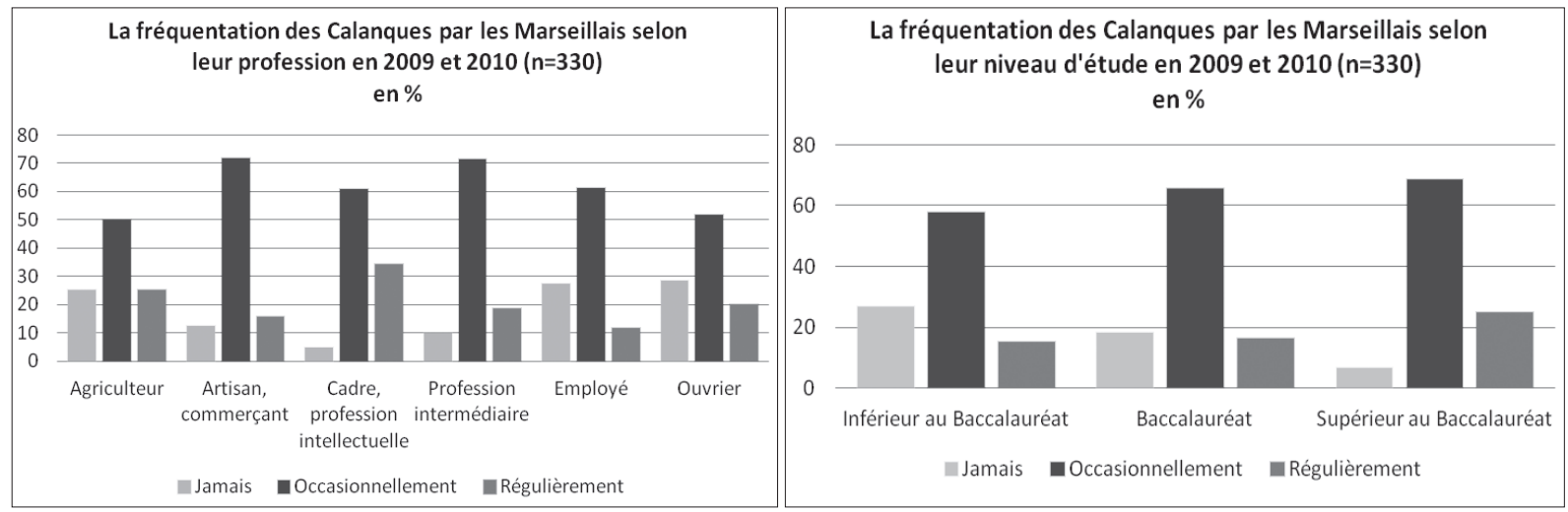

FIGURE 3 - La fréquentation des Calanques par les Marseillais: segmentation sociale.

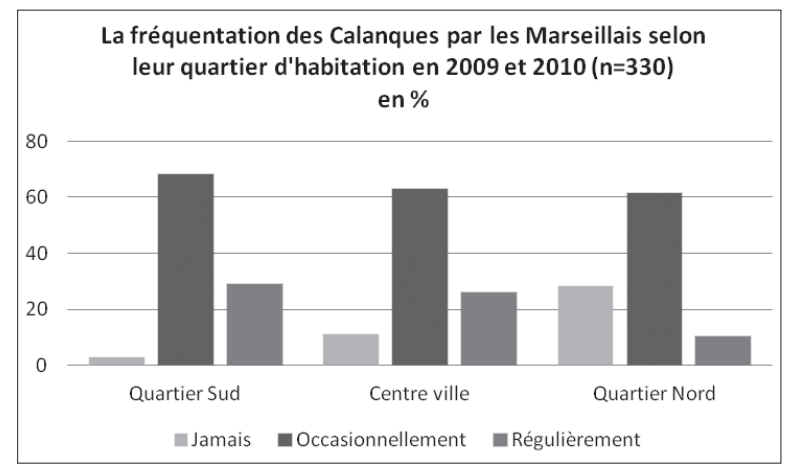

FIGURE 4 - La fréquentation des Calanques par les Marseillais: segmentation socio-spatiale.

Seule une enclave fait exception. Il s'agit du quartier de la Cayolle niché au pied du massif des Calanques et en partie construit sur l'emplacement d'un ancien camp de transit devenu bidonville, accueillant tour à tour ex-prisonniers de guerre, immigrés, victimes de la crise du logement. Malgré des politiques urbaines volontaristes menées à partir des années 1970, ce quartier demeure fortement marqué par ses origines (DELDREVE; DEBOUBT, 2011). Les habitants de la Cayolle se considèrent comme des usagers légitimes des Calanques au nom de leur proximité physique avec ce site qu'ils intègrent à leur identité territoriale. Ils dénoncent les privilèges des cabanoniers ayant droit de passage là où les barrières des routes du feu sont fermées pour les autres usagers en période estivale. En retour, les cabanoniers contestent la légitimité des "jeunes de la Cayolle", dénonçant les vols et vandalisme commis par certains d'entre eux (DELDREVE; DEBOUBT, 2011). Cette opposition prend ici des airs de lutte de classes mettant en tension des couches populaires, notamment issues de l'immigration, avec une petite bourgeoisie exécutive, se distinguant au sens bourdieusien du terme, des "jeunes de la Cayolle", tout en revendiquant un héritage populaire issu des petits pêcheurs d'antan.

Paradoxalement, se conjuguent à Marseille perméabilité physique et ségrégation sociale. Du parc public aux Calanques, de la plage au square, toutes les couches sociales marseillaises investissent abondamment les espaces verts publics et/ou les espaces naturels, à la faveur d'un climat propice. Cependant, il existe des clivages plus ou moins marqués dans le choix de ces espaces; A l'instar de la topographie marseillaise où ville et massifs s'imbriquent avec une intimité brouillant les frontières physiques entre l'urbain et le naturel, les évocations des habitants opèrent quelques chassés-croisés. Interrogés tour à tour sur leurs espaces verts et leurs espaces naturels préférés, les habitants vont de l'un à l'autre, révélant une certaine confusion lexicale, mais aussi la perméabilité des frontières physiques entre certains parcs publics et la colline (figure n. ${ }^{\circ} 5$ ). 


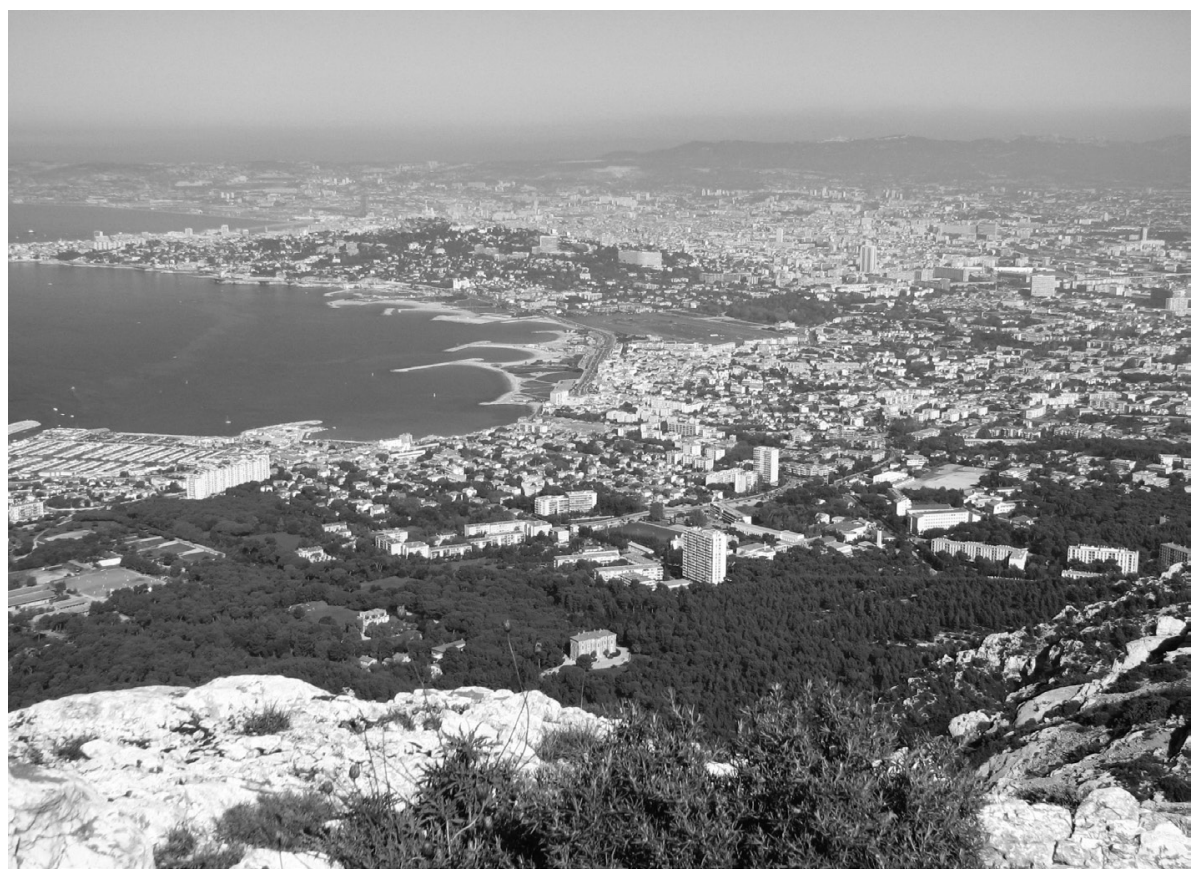

FIGURE 5 - Marseille, vue du Massif des Calanques: entre le tissu urbain et le massif périurbain, un parc public arboré, ancien domaine bastidaire.

\section{Gestion: ordonner, protéger ou jardiner la nature}

Les politiques publiques de l'aménagement du territoire ont hérité d'un regard dichotomique opposant l'urbain et le rural, l'artificiel et le naturel. Inféodée aux politiques de la ville, la gestion des parcs publics basés sur le principe d'une nature jardinée et ordonnée se tourne aujourd'hui vers des tentatives de ré-ensauvagement de la nature urbaine. Réciproquement, inféodée aux politiques de la nature, la gestion des espaces protégés, et notamment des Parcs Nationaux, se tourne aujourd'hui vers des tentatives d'intégration de l'urbanité et ses demandes croissantes de loisirs de plein air.

Marseille compte aujourd'hui 41 parcs ou jardins qui représentent une surface de 422 hectares. A côté des parcs dits "remarquables", existe une myriade de parcs de quartiers, créés notamment suite à la politique dite des mille points verts lancée par la municipalité de Gaston Deferre au début des années 1970 qui fait déclarer à ce dernier 'Qu'aucun service public ne peut abattre un arbre à Marseille sans mon autorisation personnelle". Une enquête sociologique menée auprès des agents d'entretien des jardins municipaux (BOURDIL, 2010) montre qu'une catégorisation très nette est opérée par ces agents, entre la "nature naturelle" associée aux Calanques et collines et une nature plus artificielle qu'ils contribuent à créer: “un parc, c'est un milieu naturel mais c'est nous, qui l'avons créé" (Entretien, agent). Deux catégories de parcs émanent de l'analyse des entretiens réalisés: les parcs de centre-ville, dévolus à des usages urbains de nature (promenade, jeux pour enfants, sport...), faisant l'objet d'un entretien intense et coûteux et les parcs situés en périphérie, lieux de promenades de proximité, moins entretenus et donnant lieu à une nature plus spontanée. Les parcs publics offrent ainsi une double construction: le remarquable, vitrine du savoir-faire horticole des jardiniers marseillais, localisé dans l'hyper-centre et l'ordinaire, parc de quartiers.

Actuellement, la commune tente d'intégrer une approche environnementale en mettant en place une gestion différenciée de ses espaces verts, qui consiste à laisser 
plus de place à la végétation dite spontanée, à la moindre utilisation des produits phytosanitaires et de l'eau. Cette gestion est plus facilement adaptable aux parcs de la périphérie, les moins visibles et les plus ordinaires. Mais, au nom du respect de l'environnement, un changement est peut être en train de s'opérer entre les parcs remarquables et les autres; l'ordre pourrait s'inverser, entre les parcs de périphérie dont la gestion plus écologique serait élogieuse au détriment des parcs de centre-ville coûteux en ressources naturelles et produits chimiques. Mais, ce nouvel ordre environnemental vient se heurter à plus d'un siècle d'hygiénisme urbain et de compétences horticoles dont rendent compte les attitudes différenciées des agents rencontrés (BOURDIL, 2010).

Au niveau local, ces difficultés d'adaptation transparaissent également dans les atermoiements des acteurs de l'aménagement du territoire et de l'urbanisme à l'égard de la Trame Verte et Bleue. Pensé pour favoriser le maintien de la biodiversité, cet outil prévoit de ménager des échanges faunistiques et floristiques entre des réservoirs de biodiversité, au moyen de continuités écologiques (BERGES et al., 2010). Pour ce faire, il suscite des emboîtements entre les échelles européenne, nationale, régionale et locale. Ainsi, au sein des territoires urbains, la Trame Verte et Bleue réinterroge le rapport ville/nature: alors que la nature, porteuse de multifonctionnalité, s'envisage traditionnellement comme étant au service de la ville, la ville, par voie de réciprocité, doit désormais s'envisager comme étant au service de la nature. A la faveur de cette symbiose supposée, elle devient un écosystème à part entière et doit fonctionner en tant que tel. Or, en l'absence de référentiels méthodologiques précis établis à l'échelle nationale, l'élaboration d'une Trame Verte et Bleue reste, à l'échelle locale, soumise à l'interprétation d'acteurs hétérogènes, à des modalités d'action plurielles et, de fait, à une certaine “malléabilité des politiques environnementales" (BARTHÉLÉMY; SOUCHON, 2009). A Marseille, bien que très diverses, les lectures institutionnelles de la Trame Verte et Bleue s'accordent toutes à souligner la place fondamentale qu'occupent les grands massifs naturels dans le maillage écologique marseillais. Les études liées à la réalisation simultanée du Schéma Régional de Cohérence Ecologique, du Schéma de Cohérence Territorial de la Communauté Urbaine Marseille-Provence-Métropole et du Plan Local d'Urbanisme de la commune de Marseille visent, par conséquent, à relier ces réservoirs de biodiversité grâce à des corridors écologiques intra-urbains. Or, dans ce contexte spatial, ces approches, largement portées par des acteurs de l'aménagement du territoire et de l'urbanisme encore peu rodés aux principes d'une écologie urbaine balbutiante, se heurtent aux impératifs d'une urbanisation érigée en moteur de la redynamisation territoriale. Entre le centre-ville et les massifs naturels qui circonscrivent le territoire communal, un puissant processus de densification périphérique tend alors à se surimposer sur un vaste réseau d'Espaces à Caractère Naturel potentiellement mobilisable dans un projet de trame verte intra-urbaine. Cette couronne périphérique tient, en effet, un rôle essentiel dans la structure écopaysagère marseillaise. Entre les ECN de la matrice urbaine dense (espaces centraux) et les réservoirs de biodiversité des marges naturelles (massifs), elle assure, en effet, une continuité territoriale (CONSALÈS et al., 2012) dont l'incidence écologique tend à être démontrée (LIZET et al., 2011). Territoire composite, caractérisé par des tissus urbains hétérogènes (bastides, pavillons, lotissements, grands ensembles et petits collectifs récents en résidences fermées), elle offre à voir des paysages singuliers, laissant une large part au non construit: nombreux délaissés et autres friches végétalisées hérités du passé agricole. Ceux-ci font cependant les frais du processus récent de densification périphérique. Ils mettent ainsi en exergue les injonctions paradoxales liées à une nature ordinaire (MOUGENOT, 2003), prise entre les logiques de la valorisation environnementale et celles de la consommation foncière.

A l'heure où l'urbanité s'ouvre aux désordres de la nature spontanée brouillant les frontières de l'ancien dualisme nature/culture, certains tenants de la protection de la nature semblent toujours rechercher une sorte de pureté originelle (CLAEYS; SIROST, 2010). Les inventaires servant de fondement scientifique à la création du parc national des Calanques dressent les listes des espèces autochtones et tout particulièrement endémiques, à protéger, par opposition, notamment aux espèces exotiques invasives. Sous haute surveillance scientifique, les spécimens les plus rares sont bagués, espèces remarquables d'oiseaux marins (puffin cendrés, Cormoran huppé...), mais aussi espèces végétales 
comme les pieds d'astragale et de plantin, inventoriés et cartographiés pour un suivi scientifique quasiment individualisé. Le gestionnaire se fait ici jardinier malgré lui, cultivant, selon la formule de Raphael Larrère (1994), une nature "sauvagement artificielle!". L'anthropologue Françoise Dubost s'interroge à ce propos: "Sauvegarder le sauvage, cultiver le sauvage: retour du naturel ou comble de l'artifice? Étape ultime dans l'entreprise de domestication de la nature ou règles du jeu nouvelles qui préservent la liberté du vivant?", (1997, p. 23). Mais quelle est dès lors, dans cette "nature des scientifiques" la place des usagers ordinaires?

Les postures des naturalistes les plus radicaux ayant contribué à initier le projet de Parc National des Calanques (PNC) et rêvant d'un massif majoritairement soustrait aux usagers sont nuancés aujourd'hui par les nouvelles politiques de protection de la nature. Le passage d'une protection qui ne se limite plus seulement aux "espaces naturels sensibles particulièrement remarquables" (loi du 22 juillet 1960), mais s'étend plus largement à "un riche patrimoine naturel, culturel et paysager" impulsé par la réforme du cadre juridique (loi du 14 avril 2006) régissant la création et la gestion des Parcs Nationaux de France (PNF) marque la volonté d'une articulation entre nature et culture. Il ne s'agirait dès lors plus de "soustraire la nature à l'influence humaine" (LEPART; MARTY, 2006), mais d'institutionnaliser un patrimoine socio-naturel, désignant par là des objets hybrides échappant à la traditionnelle dichotomie nature/culture.

Le Parc National des Calanques est le premier de cette nouvelle génération de PNF. Il endosse à bien des égards un statut de précurseur. Outre le cadre juridique nouveau, il s'agit aussi du premier PNF situé aussi prêt d'une agglomération de grande taille, et du premier parc littoral incluant des espaces terrestres et marins. Dans ce contexte et avec l'implication de nouvelles générations de scientifiques et de gestionnaires formés à la gestion intégrée, le Parc National des Calanques s'est donné pour slogan: "la Nature en partage".

Ce slogan aux airs consensuels a une histoire tourmentée. Les premières actions militantes des associations d'usagers (manifestation contre l'extension d'une carrière dans les Calanques de Port-Pin, Port-Miou et En-Vau en 1910), la demande de création d'un Parc Na- tional des Calanques adressée dès 1971 (DELDREVE; DEBOUBT, 2011), la création du GIP (Groupement d'Intérêt Professionnel) des Calanques en 1999 en vue de préfigurer le futur Parc National, les manifestations et les pétitions pour ou contre la création du PNC, et enfin l'organisation d'une longue concertation en 2010, marquent plusieurs décennies de controverses animées. Si les protagonistes s'accordent majoritairement sur la nécessité d'extraire le massif des Calanques à l'activité industrielle et la pression immobilière, en revanche, la question de la régulation de la fréquentation récréative fait débat. Face aux gestionnaires qui déploient des dispositifs techniques et/ou réglementaires (barrières, balisages, limitation/interdiction d'accès) afin de canaliser, sinon réguler la fréquentation du massif, des usagers mécontents s'inquiètent de voir, avec la création du PNF, se renforcer de telles politiques, à leur détriment, comme le révèle les réactions recueillies lors de l'enquête sur site par entretiens semi-directifs. Ce promeneur regrette: "interdire, ce serait complètement dommage. Peut-être les surveiller un peut plus [...] Mais je pense que ce serait dommage de les interdire complètement". Ce pêcheur à pied inquiet, s'insurge: "La pierre angulaire du Parc, c'est la pêche [...] c'est une catastrophe. Dans le projet qu'on a vu d'interdire la pêche à partir de là jusqu'au Frioul c'est une aberration".

Les usagers des Calanques sont toutefois particulièrement difficiles à quantifier. Le nombre et la dispersion des points d'entrée dans le massif, la diversité des usages, mais aussi l'absence jusqu'en 2011 d'une politique coordonnée d'observation de la fréquentation prêtent le flanc aux spéculations idéologiques en lieu et place d'une connaissance et d'une quantification objective de la fréquentation. Des données disparates existent, mais la diversité des protocoles d'observation dont elles sont issues limite les comparaisons dans le temps et l'espace. Ces sources hétérogènes, permettent d'identifier la diversité des usages des Calanques et d'en proposer une hiérarchie générale, sans toutefois autoriser une quantification générale robuste et moins encore une quantification de leur évolution dans le temps. La quantification des campagnes d'information et de sensibilisation réalisées en période estivale par les patrouilleurs du GIP-Calanques fournissent quelques ordres de grandeurs. Toutefois, les variations annuelles 
observées tiennent à de nombreuses variables non contrôlées, telles que la météo, le nombre de journées de fermeture du massif en cas de risque incendie et surtout, le nombre de patrouilleurs en fonction et la diversité des tâches qui leurs sont attribuées.

Ce déficit de données quantitatives et longitudinales fiables nourri des débats passionnés sur le sujet. Ainsi, alors que certains gestionnaires tendent à développer des discours dramatisant, basés sur une peur de la sur-fréquentation, fantasmant des hordes d'urbains à l'assaut d'une nature sauvage en danger, certains discours journalistiques ${ }^{4}$ idéalisent une nature sans barrière sociale, où tous les Marseillais, même les plus modestes auraient un accès démocratisé à cet espace de nature exceptionnel. Les condamnations naturalistes de la société moderne productrice de sur-fréquentation et son corollaire qu'est l'idéalisation du passé amène à s'interroger sur la nouveauté du phénomène. Les rares rétrospectives historiques sont en effet à ce titre éloquentes. Comme en témoignent les recherches ethnographiques et historiques de C. Gontier (1991), la sur-fréquentation de certaines Calanques, notamment celles très tôt desservies par navettes et bateaux taxis, était dénoncée par les protagonistes les plus critiques dès le début du XXème siècle. Il n'en reste pas moins, qu'elle soit objectivée ou non ou bien seule fait de peurs contemporaines hautement symboliques (CLAEYS; SIROST, 2010), cette (sur)fréquentation est au cœur des politiques de protection de la nature qui prennent la forme de gestion de flux d'usagers, circulant entre ville et collines, brouillant les anciennes taxonomies (urbain/ rural, sauvage/domestique).

\section{Conclusion: tous jardiniers de la nature!}

L'expérience marseillaise révèle de façon exacerbée une double évolution de la gestion de la nature. $\mathrm{Au}$ moment où les jardiniers opèrent un assouplissement de leur contrôle de la nature ordonnée, les gestionnaires des espaces "naturels" tendent à déployer des politiques à l'interventionnisme accru. La nature urbaine prend ses aises et s'affiche, invitée en cela par quelques ges- tionnaires pionniers, inspirés par les nouveaux discours scientifiques rompant avec l'historique urbaphobie des naturalistes (BAUBÉROT; BOURILLON, 2009); tandis que les gestionnaires d'espaces protégés cèdent au paradoxe de la nature sauvage aménagée (LARRÈRE, 1994; DUBOST, 1997; CLAEYS-MEKDADE; JACQUÉ, 2000; KALAORA, 2001), tiraillés entre leur rôle d'accueil d'un public croisant et leur volonté de conservation des écosystèmes.

De tels processus prennent forme aujourd'hui à travers deux projets mettant la nature au cœur des préoccupations métropolitaines marseillaises: l'élaboration d'une trame verte et la création du parc national des Calanques, relevant d'une institutionnalisation du dialogue entre logiques urbaine et écologique. A ce titre, la résistance de l'ancienne dichotomie entre ville et nature est au cœur des débats suscités par ces projets, questionnant les sphères de compétences et de pouvoir traditionnellement en charge de la gestion urbaine d'un côté et de l'autre de la gestion des écosystèmes. Dans quelle mesure les acteurs et décideurs de la ville laisseront les naturalistes s'immiscer dans la gestion de l'espace urbain? Réciproquement, quel est le seuil de tolérance des milieux "naturels", mais aussi, sinon d'abord de leurs gestionnaires, face à la fréquentation urbaine des Calanques? Ainsi, la possible mise en œuvre d'une gestion environnementale du rapport ville/nature dépend au final d'affaires humaines, traversées depuis toujours par des rapports de pouvoir.

A défaut de clore les querelles entre ville et nature, ces recompositions des territoires et de leurs modes de gestion ouvrent d'autres disputes concernant l'attribution de ce nouveau statut de "jardinier de la nature". Ici, la notion d'écologie urbaine ne serait plus seulement une métaphore, mais rendrait compte, enfin, d'une réalité matérielle et sociale en construction où l'obsolète frontière entre nature et artifice laisserait la place, à un système socio-naturel "assumé", comme nous y invite Jean-Pierre Le Dantec: “A l'heure où l'urbain généralisé supplante le couple traditionnel ville/campagne, nous avons à créer de nouveaux jardins bien sûr; mais nous devons aussi, et peut être surtout, entretenir artificiel-

${ }^{4}$ Voir notamment le numéro thématique de la revue Géo (mars, 2011). 
lement (c'est-à-dire comme des jardins) les territoires voués à la friche (agricole ou industrielle); c'est à ce prix que ne disparaîtront ni les paysages les plus proches de l'état sauvage, ni ceux créés par l'homme dans le temps long de la civilisation rurale, puis le temps court de la civilisation industrielle. Mais, ce faisant, nous devons être conscients que, dès lors, une mutation civilisationnelle majeure est en cours. Dans cette hypothèse en effet, c'est la troisième nature (le jardin) qui devient, en tant que mémoire et espace expérimental, le modèle

\section{Bibliographie}

AZIBI, L.; PARMASKYAN, P. Comprendre le Massif des Calanques. De la pratique au "concernement": Cabanonniers et Randonneurs. Mémoire de Master MASS, Université Aix-Marseille, 2006.

BARTHÉLÉMY, C.; SOUCHON, Y. La restauration écologique du Rhône sous le double regard du sociologue et de l'écologue. Natures Sciences Sociétés, n. 17, p. 113-121, 2009.

BAUBÉROT, A.; BOURILLON, F. Urbaphobie. La détestation de la ville aux XIX et XX siècles. BAUBEROT; A.; BOURILLON, F. (Dir.). Bordeaux: Ed. Bière, 2009.

BERGES, L.; ROCHE, P.; AVON, C. Corridors écologiques et conservation de la biodiversité: intérêts et limites pour la mise en place de la Trame verte et bleue. Sciences, Eaux et Territoires, IRSTEAU, n. 3, p. 34-39, 2010.

BLANC, N.; CLERGEAU, P. Installer une trame verte dans la ville. Revue Urbanisme, Hors-Série, Enjeux et Débats, 2010.

BOURDIL, C. Approche socio-écologique des relations entre gestion et biodiversité des parcs publics à Marseille. Rapport de stage, LPED, Master 1 Ingénierie Ecologique et Gestion de la Biodiversité, 2010.

BROMBERGER, C. Droit au but. Images et imaginaire de Marseille à travers son football. Marseille, v. 159, p. 50-55, 1991.

CESARI, J.; MOREAU, A.; SCHLEYER-LINDENMANN, A. Plus Marseillais que moi tu meurs. Migrations identités et territoires à Marseille. L'Harmattan, 2001.

CHALVET. M. L'invention de la forêt méditerranéenne de la fin du XVIII siècle aux années 1960. Thèse de doctorat d'Histoire, Université de Provence, 2001.

CHANCEL, J. M.; BORRUEY, R. La maison des villes et la maison des champs, la bastide marseillaise. Méditerranée - Re- d'une première et d'une seconde nature imitées: le parc "naturel", le "paysage protégé", qui en viennent à constituer une "nature" inédite, une nature artefact au statut proche de celui de Lascaux bis vis-à-vis de son modèle invisible" (2003, p. 14). Telle peut être une des lectures de la problématique environnementale: ne plus tenter en vain de discerner nature et culture mais assumer leur imbrication et viser leur complémentarité (DESCOLA, 2005).

vue Géographique des Pays Méditerranéens, Aix-en-Provence, n. 1-2, p. 81-84, 1993.

CLAEYS, C. Créer un parc national des Calanques: préserver, partager ou confisquer un patrimoine socio-naturel? Colloque "Patrimonialiser la nature: valeur et processus", SET, Université de Pau, 7-9 septembre, Actes à paraitre, 2011.

(Dir.). Iles et Calanques de Marseille: discours, pratiques et attentes des marseillais. Atelier Méthodologique du Master MASS, Promotions 2008-2010, Université de la Méditerranée, 2010.

; SIROST, O. Proliférantes Natures, numéro thématique de la revue Etudes rurales, n. 185, Juin-Juillet 2010.

CLAEYS-MEKDADE, C.; JACQUÉ, M. Les fonctions “nonmarchandes" d'une forêt méditerranéenne: loisir et détente sur le massif de la Clape. Revue Forêt méditerranéenne, T. XXI, n. 1, p. 19-24, 2000.

CLERGEAU, P. Une écologie du paysage urbain. Paris: Éditions Apogée, 2007. 136 p.

CONSALÈS, J-N. Les jardins familiaux de Marseille, Gênes et Barcelone: entre enjeux potentiels et fonctions réelles de l'agriculture urbaine. Rives Méditerranéennes, UMR 6570 Telemme / MMSH, Aix-en-Provence, n. 15, p. 21-39, 2003.

. Les jardins familiaux à Marseille, Gênes et Barcelone: laboratoires territoriaux de l'agriculture urbaine dans l'Arc Méditerranéen. Thèse de doctorat en Géographie et aménagement du territoire réalisée à l'Université de Provence et au sein de l'UMR TELEMME, sous la direction de Madame le Professeur Claudine Durbiano (Université de Provence). Aix-en-Provence, le $17 / 12 / 2004.555$ p. 
Jardins familiaux et développement durable: entre discours théoriques et actes concrets. In: DA LAGE, A.; AMAT, J. P.; FRÉROT, A. M.; GUICHARD-ANGUIS, S.; JULIEN-LAFERRIERE, B.; WICHEREK, S. P. (Dir.). L'Après Développement Durable, Espaces, Nature, Culture et Qualité. Lonrai: Ellipses, 2008. p. 203-211.

; GOIFFON, M. Le massif des Calanques (Marseille-Cassis) et la Pointe des Châteaux (Saint-François, Guadeloupe). Périmètres d'intervention et mesures de protection sur deux grands sites littoraux périurbains. Méditerranée - Revue Géographique des Pays Méditerranéens, Aix-en-Provence, $\mathrm{n}$. 3-4, p. 29-35, 2005.

et al. La friche comme révélateur de la "schizophrénie du vert" à Marseille. $135^{\mathrm{ème}}$ Congrès National des Sociétés savantes et scientifiques. Neuchâtel, 10 avril 2010.

CORBIN, A. (Dir.). L'avènement des loisirs, Paris: Aubier, 1995.

D.D.A. Agglomération marseillaise, étude de l'agriculture en milieu urbain. Une étude de l'Atelier Départemental d'Etudes et d'Aménagement Rural de la Direction Départementale de l'Agriculture, $1971.79 \mathrm{p}$.

DEBOUBT, P. (Dir.). Inégalités écologiques, territoires littoraux et développement durable. Villeneuve d'Asq: Septentrion Presses Universitaires, 2010. 409 p.

DELDREVE, V.; DEBOUBT, Ph. (Dir.). Un Parc National pour les Calanques de Marseille? Construction territoriale, forme de concertation et principes de légitimité. APR CDE 2 $d u$ MEDDT, 2011. $328 \mathrm{p}$.

DESCOLA, Ph. Par de-là nature et culture. Paris: Gallimard, 2005.

DUBOST, F. Les jardins ordinaires. Paris: L'Harmattan, 1997.

; LIZÉE, B. La nature dans la cité, de l'hygiénisme au développement durable, Bienfaisantes natures. Communications, v, 74, p. 5-18, 2003.

DUMAZEDIER, J. Révolution culturelle du temps libre 19681988. Paris: Meridiens Klincksieck, 1988.

DURBIANO, C. De la marginalisation à la reconquête environnementale et patrimoniale des collines de l'aire métropolitaine marseillaise. Rives Méditerranéennes, n. 15, p. 41-52, 2003.

GIP CALANQUES. 2009, Etude de fréquentation terrestre et marine, 2009. Disponible in: <www.gipCalanques.fr>.

GONTIER, C. Le cabanon marseillais. Images et pratiques. Marseille: Cerfise, 1991.
HÉRAT, A. L'amènagement des plages du Prado à Marseille: la construction d'un bien commun. In: DEBOUBT, Ph. (Dir.). Inégalités écologiques, territoires littoraux et développement durable. Villeneuve d'Ascqu (France): Presse Universitaires du Septentrion, 2010. p. 223-236.

KALAORA, B. A la conquête de la pleine nature. Ethnologie Française, n. 2001/2 - Tome XXXVII, p. 591-597, 2001.

LANASPEZE, B. Marseille ville sauvage, essai d'écologie urbaine. Marseille: Actes Sud, 2012. 198 p.

LARRÈRE, R. Sauvagement Artificiel. Le Courrier de l'environnement de l'Inra, v. 21, p. 35-37, 1994.

LE DANTEC, J-P. Jardins et paysages, textes critiques de l'antiquité à nos jours. Paris: Editions de la Villette, 2003. 635 p.

LEPART, J.; MARTY, P. Des réserves de nature aux territoires de la biodiversité. L'exemple de la France. Annales de Géographie, v. 5, n. 651, p. 485-507, 2006.

LIMIDO, L. L'art des jardins sous le Second Empire, Jean-Pierre Barillet Deschamps (1824-1873). Collection Pays/ Paysages, Champ Vallon, Floch à Mayenne, 2002. 281 p.

LIZÉE, M.-H.; MAUFFREY, J.-F.; TATONI, T.; DESCHAMPS-COTTIN, M. Monitoring urban environments on the basis of biological traits. Ecological Indicators, n. 11, p. 353-361, 2011.

MATHIEU, N.; GUERMOND Y., La ville durable, du politique au scientifique, Paris : Editions Quae, 2005, 286 p.

MASSENA-GOURC, G. La protection à l'épreuve de la diversité des usages, le massif des Calanques. Etudes Rurales, n. 133-134, p. 149-162, 1994.

MERY, J. Marseille et les Marseillais. Paris: A. Bourdillat et $\mathrm{C}^{\mathrm{e}}$ éditeur, $1860.309 \mathrm{p}$.

MOUGENOT, C. Prendre soin de la nature urbaine. Paris: Editions de la Maison des Sciences de l'Homme, Institut National de la Recherche Agronomique, 2003. 230 p.

NIEL, A. Le parc balnéaire du Prado de Marseille: exemple d'une territorialisation de l'espace par ses usagers sportifs. In: SIROST, O. (Dir.). La vie au grand air. Aventures du corps et évasions vers la nature. Nancy: Presses Universitaires de Nancy. 2009. p. 237-252.

RIANI, A. Une autre ville: les bastides. Marseille Revue Culturelle, Marseille, n. 192, p. 6-11, 2000.

RONCAYOLO, M. Les Grammaires d'une ville. Essai sur la genèse des structures urbaines à Marseille. Paris: Éditions de l'EHESS, 1996. 507 p. 
SANSOT, P. Jardins Publics. Paris: Payot \& Rivages, 1995.

TATONI, T.; LE MIRE PECHEUX, L. (Dir.) Caractérisation de la fréquentation littorale et détermination d'indicateurs de vulnérabilité écologique pour définir des modes de gestion durable - Cas de la bande littorale du projet de Parc National des Calanques, Rapport Intermédiaire, Programme de Recherche LITEAU du Ministère de l'Ecologie, 2010.

VERGES, P.; PRUNEAU, V. Vivre à Marseille: Marseille vue par les Marseillais. In: LANGEVIN, P. (Dir.); JUAN, J-C. Paris: Ed. La Documentation Française, 2007. p. 119-126.
VIARD, J. Le marin et le laboureur. Marseille, Histoires de Famille, Série Monde, Hors Série n. 36, Autrement, p. 25-36, 1989.

VIDAL-NAQUET, P. Marseille: les ruisseaux, le canal et la mer. Paris: Editions L'Harmattan, 1993. 158 p.

WEBER, F. L'honneur des jardiniers, les potagers dans la France du XXe siècle. Collection Socio-Histoires, 1998. 287 p.

Recebido em 19 de junho de 2012. Aceito em 23 de outubro de 2012. Publicado em dezembro de 2012. 